EFI-03-41

hep-th/0308172

\title{
Comments on Half S-Branes
}

\author{
Kazumi Okuyama \\ Enrico Fermi Institute, University of Chicago \\ 5640 S. Ellis Ave., Chicago IL 60637, USA \\ kazumi@theory.uchicago.edu
}

Following hep-th/0305177, we write the boundary state of half S-brane in bosonic string theory as a grand canonical partition function of a unitary matrix model. From this representation, it follows that the annulus amplitude can be written as a grand canonical partition function of a unitary two-matrix model. We also show that the contribution of the exponentially growing couplings to the timelike oscillators can be resummed in a certain annulus amplitude.

August 2003 


\section{Introduction}

The decay of unstable D-brane is an interesting laboratory to study various properties of string theory in time-dependent backgrounds [1 20]. Moreover, the study of unstable D-brane will give us a new perspective to the open/closed duality [21,22]. In this paper, we consider the decay of D-brane in bosonic string theory described by the timelike boundary Liouville theory:

$$
S_{\mathrm{TBL}}=-\frac{1}{2 \pi} \int_{\Sigma} \partial X^{0} \bar{\partial} X^{0}+\lambda \oint_{\partial \Sigma} e^{X^{0}}
$$

which is also referred to as the half S-brane. Throughout this paper, we set $\alpha^{\prime}=1$. This negative norm boson theory can be defined by an analytic continuation of the spacelike boundary Liouville theory.

There are two different approaches to define this analytic continuation. In the first approach, we start with the spacelike Liouville theory with background charge $Q=b+b^{-1}$ and take the limit $b \rightarrow i$ [11]. In this approach, the half S-brane boundary state is obtained by the analytic continuation of the boundary state in the spacelike Liouville theory, which describes the D-brane extended in the Liouville direction [23],

$$
\left.\left|B_{\mathrm{LFZZ}}\right\rangle=\int_{-\infty}^{\infty} d E \lambda^{-i E} \frac{\pi}{\sinh \pi E}|E\rangle\right\rangle .
$$

We call this state the "Lorentzian FZZ state." This is expanded in the Ishibashi state $|E\rangle\rangle$ built on the bulk primary operator $e^{i E X^{0}}$. In the second approach, which is adopted in the Sen's original paper [2], we perform an inverse Wick rotation of the boundary state of a spacelike $c=1$ boson $X$ with the boundary interaction $e^{i X}$ [24,25, 10, 11]:

$$
\left.|B\rangle=\sum_{j} \sum_{m \geq 0}^{j}\left(\begin{array}{c}
j+m \\
2 m
\end{array}\right)(i \lambda)^{2 m}|j, m, m\rangle\right\rangle .
$$

In this case, the boundary state is expanded in terms of the Ishibashi state built on the discrete states. Note that in these two approaches we analytically continue from the spacelike CFTs with different central charges: $c \neq 1$ for (1.2) and $c=1$ for (1.3).

It is important to notice that the resulting Lorentzian boundary states (1.2) and (1.3) are different. In particular, the exponentially growing couplings to the timelike oscillators [13] in (1.3) are not included in (1.2). Mathematically, this reflects the fact that the analytic continuation to define (1.1) is not unique. Physically, as discussed in [11, 16, 19, 26, 27, 21], in time-dependent backgrounds the worldsheet CFT has ambiguities corresponding to the 
choices of incoming state, or vacuum. Therefore, we should regard these two different boundary states (1.2) and (1.3) as a result of the two different choices of the vacuum.

Most of the computations of Lorentzian annulus amplitude considered in the literature are based on the Lorentzian FZZ state (1.2). Therefore, it is natural to ask what happens if we use (1.3) in the computation of annulus amplitude. However, the exponentially growing couplings to the massive modes found in [13] make this very difficult, both technically and conceptually. It was shown [13] that the coupling to the timelike oscillator at a fixed level $n \geq 2$ in (1.3) grows exponentially at late times, and we cannot resum these terms as opposed to the situation at level 0 and 1 . However, there is a possibility to perform a resummation over the terms at different levels. The recent result of [18 will be potentially useful for this purpose, because it provides us an efficient way to compute the coefficient of (1.3) in terms of the unitary matrix integral. In this paper, we will show that the contribution of those exponentially growing terms can be resummed in a certain annulus amplitude.

This paper is organized as follows. In section 2, after reviewing the result of [18, we write the boundary state (1.3) as a grand canonical partition function of a unitary matrix model. We also obtain a useful decomposition of $|B\rangle$, in which the first term corresponds to $\left|B_{\mathrm{LFZZ}}\right\rangle$ and the second term represents the exponentially growing coupling to the timelike oscillators [13]. In section 3, we write the annulus amplitude between two $|B\rangle$ 's as a grand canonical partition function of a unitary two-matrix model, and present some examples of the matrix integral. In section 4 , we consider the annulus amplitude between $|B\rangle$ and the first term in the decomposition of $|B\rangle$ obtained in section 2. We show that in this amplitude we can perform a resummation of the exponentially growing timelike oscillators. Section 5 is devoted to discussions. In appendix A, we give a proof of some identity used in section 4.

\section{Half S-brane Boundary State}

In this section, we consider the boundary state $|B\rangle$ of timelike boundary Liouville theory (1.1). Following [18], we write $|B\rangle$ as a grand canonical partition function of a unitary matrix model.

In this paper, we use the following notation for a partition $\mu=\left(n_{1}^{N_{1}} \cdots n_{k}^{N_{k}}\right)$ :

$$
|\mu|=\sum_{i} n_{i} N_{i}, \quad \ell(\mu)=\sum_{i} N_{i}, \quad z_{\mu}=\prod_{i} n_{i}^{N_{i}} N_{i} !,
$$

i.e., $|\mu|, \ell(\mu)$, and $z_{\mu}$ denote the total number, the length, and the norm of the partition $\mu$, respectively. $n_{i}$ 's are strictly increasing: $n_{1}<\cdots<n_{k}$. 


\subsection{Boundary State and Unitary Matrix Integral}

To write the boundary state as a matrix integral, we first review the result of [18]. It was shown in [18] that the disk one-point function $\left\langle{ }_{0}^{\circ} V^{(\sigma ; \tilde{\sigma}){ }_{\circ}^{\circ}}\right\rangle$ of the operator

$$
V^{(\sigma ; \tilde{\sigma})}=\frac{1}{\sqrt{z_{\sigma} z_{\tilde{\sigma}}}} \prod_{i, j}\left(\frac{\sqrt{2}}{\left(n_{i}-1\right) !} \partial^{n_{i}} X^{0}\right)^{N_{i}}\left(\frac{\sqrt{2}}{\left(\tilde{n}_{j}-1\right) !} \bar{\partial}^{\tilde{n}_{j}} X^{0}\right)^{\widetilde{N}_{j}}
$$

labeled by $\sigma=\left(n_{i}^{N_{i}}\right)$ and $\widetilde{\sigma}=\left(\tilde{n}_{i}^{\tilde{N}_{i}}\right)$, is given by the unitary matrix integral

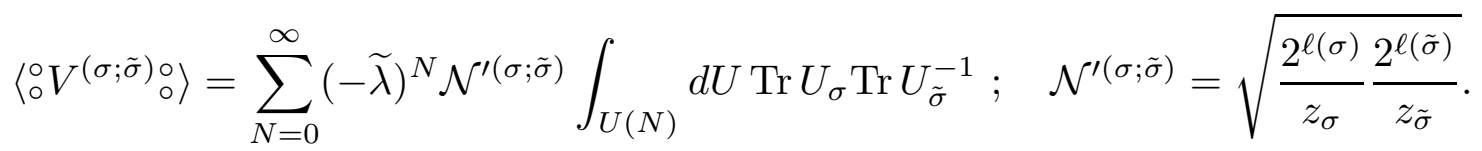

The measure $d U$ is normalized so that $\int_{U(N)} d U=1$. This remarkable relation (2.3) was obtained by expanding the boundary interaction in terms of the "time-dependent coupling" 1

$$
\widetilde{\lambda}=\lambda e^{x^{0}}
$$

and then performing the path integral over the non-zero modes of $X^{0} . x^{0}$ in $\tilde{\lambda}$ is the zeromode of the worldsheet boson $X^{0}$. The rank $N$ of group $U(N)$ is the number of insertions of tachyon operator, and the $N$ eigenvalues of $U$ describes the position of the $N$ tachyon operators on the unit circle, which is the boundary of the disk worldsheet. The operator $V^{(\sigma ; \tilde{\sigma})}$ in $(2.2)$ corresponds to the state

$$
|\sigma, \tilde{\sigma}\rangle=\frac{1}{\sqrt{z_{\sigma} z_{\tilde{\sigma}}}} \alpha_{-\sigma} \widetilde{\alpha}_{-\tilde{\sigma}}|0\rangle
$$

under the mapping rule

$$
\alpha_{-n} \leftrightarrow \frac{\sqrt{2} i}{(n-1) !} \partial^{n} X=\frac{\sqrt{2}}{(n-1) !} \partial^{n} X^{0}
$$

In (2.3) and (2.5), we used the abbreviation

$$
\alpha_{-\sigma}=\prod_{i} \alpha_{-n_{i}}^{N_{i}}, \quad \operatorname{Tr} U_{\sigma}=\prod_{i}\left(\operatorname{Tr} U^{n_{i}}\right)^{N_{i}}
$$

The normalization of $|\sigma, \tilde{\sigma}\rangle$ is chosen so that $\left\langle\sigma, \tilde{\sigma} \mid \sigma^{\prime}, \tilde{\sigma}^{\prime}\right\rangle=\delta_{\sigma, \sigma^{\prime}} \delta_{\tilde{\sigma}, \tilde{\sigma}^{\prime}}$. In (2.5), the oscillators $\alpha_{n}$ should be inverse Wick rotated $\alpha_{n} \rightarrow-i \alpha_{n}^{0}$ when we talk about the boundary

1 In [18], $\lambda$ and $\widetilde{\lambda}$ were denoted as $g$ and $\widetilde{g}$, respectively. 
state of the timelike Liouville theory. But we continue using the Euclidean notation $\alpha_{n}$ in order to avoid the appearance of $i$ 's in equations.

The ordering $\stackrel{\circ}{\circ} \circ$ o appearing in $\left\langle{ }_{0}^{\circ} V^{(\sigma ; \tilde{\sigma})}{ }_{\circ}^{\circ}\right\rangle$ is the boundary normal ordering defined by the Green function on the disk including the effect of the image charge. On the other hand, the overlap between the boundary state $|B\rangle$ and the state $|\sigma, \tilde{\sigma}\rangle$ is given by the disk amplitude $\left\langle: V^{(\sigma ; \tilde{\sigma})}:\right\rangle$ computed with respect to the bulk normal ordering $: \bullet$ These two normal orderings are related by

$$
: X^{0}(z, \bar{z}) X^{0}(w, \bar{w}):={ }_{\circ}^{\circ} X^{0}(z, \bar{z}) X^{0}(w, \bar{w})_{\circ}^{\circ}+\log |z \bar{w}-1| .
$$

The relation between the two amplitude $\left\langle: V^{(\sigma ; \tilde{\sigma})}:\right\rangle$ and $\left\langle{ }_{\circ}^{\circ} V^{(\sigma ; \tilde{\sigma}){ }_{\circ}^{\circ}}\right\rangle$ can be written in a compact form in terms of the states

$$
|B\rangle=\sum_{\sigma, \tilde{\sigma}}\left\langle\bullet V^{(\sigma ; \tilde{\sigma})} \bullet\right\rangle|\sigma, \tilde{\sigma}\rangle, \quad|A\rangle=\sum_{\sigma, \tilde{\sigma}}\left\langle{ }_{\circ}^{\circ} V^{(\sigma ; \tilde{\sigma}) \stackrel{\circ}{\circ}\rangle}\right\rangle|\sigma, \tilde{\sigma}\rangle
$$

By noticing the relation

$$
: \frac{\sqrt{2}}{(n-1) !} \partial^{n} X^{0}(0) \frac{\sqrt{2}}{(m-1) !} \partial^{m} X^{0}(0):=\stackrel{\circ}{\circ} \frac{\sqrt{2}}{(n-1) !} \partial^{n} X^{0}(0) \frac{\sqrt{2}}{(m-1) !} \partial^{m} X^{0}(0)_{\circ}^{\circ}-n \delta_{n, m},
$$

and the correspondence rule (2.6), it is easy to see that $|B\rangle$ and $|A\rangle$ are related by $⿴$

$$
|B\rangle=\exp \left(-\sum_{n=1}^{\infty} \frac{1}{n} \alpha_{-n} \widetilde{\alpha}_{-n}\right)|A\rangle .
$$

Plugging (2.3) into (2.9), and performing the summation over $\sigma$ and $\tilde{\sigma},|A\rangle$ is written as

$$
|A\rangle=\sum_{N=0}^{\infty}(-\widetilde{\lambda})^{N} \int_{U(N)} d U \exp \left[\sum_{n=1}^{\infty} \frac{\sqrt{2}}{n}\left(\operatorname{Tr} U^{n} \alpha_{-n}+\operatorname{Tr} U^{-n} \widetilde{\alpha}_{-n}\right)\right]|0\rangle .
$$

Finally, using the relation (2.11) between $|A\rangle$ and $|B\rangle$, we arrive at the main result of this section:

$$
\begin{aligned}
|B\rangle & =\sum_{N=0}^{\infty}(-\widetilde{\lambda})^{N} \int_{U(N)} d U \exp \left[\sum_{n=1}^{\infty} \frac{1}{n}\left(-\alpha_{-n} \widetilde{\alpha}_{-n}+\sqrt{2} \operatorname{Tr} U^{n} \alpha_{-n}+\sqrt{2} \operatorname{Tr} U^{-n} \widetilde{\alpha}_{-n}\right)\right]|0\rangle \\
& \equiv \sum_{N=0}^{\infty}(-\widetilde{\lambda})^{N} \int_{U(N)} d U\left|B_{U}\right\rangle,
\end{aligned}
$$

2 See e.g. eq.(2.7.14) in [28]. This derivation is due to F. Larsen. We would like to thank N. Constable and F. Larsen for discussion on this point. 
where $\left|B_{U}\right\rangle$ is the coherent state obeying

$$
\left(\alpha_{n}+\widetilde{\alpha}_{-n}\right)\left|B_{U}\right\rangle=\sqrt{2} \operatorname{Tr} U^{n}\left|B_{U}\right\rangle .
$$

Note that in the Euclidean theory the momentum is proportional to the rank of $U(N)$

$$
\alpha_{0}=\widetilde{\alpha}_{0}=\sqrt{\frac{\alpha^{\prime}}{2}} p=\frac{\sqrt{2}}{2} \operatorname{Tr} \mathbf{1}=\frac{1}{\sqrt{2}} N
$$

To summarize, the boundary state of timelike Liouville theory is the grand canonical partition function of the unitary matrix integral (2.13). Here, the zero-mode $x^{0}$, or time, plays the role of the chemical potential for $N$. 3

Another useful expression of $|B\rangle$ can be obtained by expanding the matrix integral in terms of the characters $\chi_{Y}(\sigma)$ of symmetric group. Using the Frobenius relation, the matrix integral is written as

$$
\int_{U(N)} d U \operatorname{Tr} U_{\sigma} \operatorname{Tr} U_{\tilde{\sigma}}^{-1}=\sum_{\ell(Y) \leq N} \chi_{Y}(\sigma) \chi_{Y}(\tilde{\sigma})=z_{\sigma} \delta_{\sigma, \tilde{\sigma}}-\sum_{\ell(Y)>N} \chi_{Y}(\sigma) \chi_{Y}(\tilde{\sigma})
$$

Here we used the completeness relation $\sum_{Y} \chi_{Y}(\sigma) \chi_{Y}(\tilde{\sigma})=z_{\sigma} \delta_{\sigma, \tilde{\sigma}}$. Plugging (2.16) into (2.3), $|A\rangle$ is rewritten as

$$
\begin{aligned}
|A\rangle= & \sum_{N=0}^{\infty}(-\tilde{\lambda})^{N} \sum_{\sigma, \tilde{\sigma}} \mathcal{N}^{\prime(\sigma, \tilde{\sigma})} \sum_{\ell(Y) \leq N} \chi_{Y}(\sigma) \chi_{Y}(\tilde{\sigma})|\sigma, \tilde{\sigma}\rangle \\
= & f \exp \left(2 \sum_{n=1}^{\infty} \frac{1}{n} \alpha_{-n} \tilde{\alpha}_{-n}\right)|0\rangle-\sum_{N=0}^{\infty}(-\tilde{\lambda})^{N} \sum_{\sigma, \tilde{\sigma}} \mathcal{N}^{\prime(\sigma, \tilde{\sigma})} \sum_{\ell(Y)>N} \chi_{Y}(\sigma) \chi_{Y}(\tilde{\sigma})|\sigma, \tilde{\sigma}\rangle \\
= & (f-1) \exp \left(2 \sum_{n=1}^{\infty} \frac{1}{n} \alpha_{-n} \tilde{\alpha}_{-n}\right)|0\rangle+|0\rangle \\
& \quad-\sum_{N=1}^{\infty}(-\tilde{\lambda})^{N} \sum_{\sigma, \tilde{\sigma}} \mathcal{N}^{\prime(\sigma, \tilde{\sigma})} \sum_{\ell(Y)>N} \chi_{Y}(\sigma) \chi_{Y}(\tilde{\sigma})|\sigma, \tilde{\sigma}\rangle .
\end{aligned}
$$

In the third line, we rewrote the $N=0$ term by using

$$
\sum_{\ell(Y)>0} \chi_{Y}(\sigma) \chi_{Y}(\tilde{\sigma})=\delta_{\sigma, \tilde{\sigma}} z_{\sigma}-\delta_{\sigma, 0} \delta_{\tilde{\sigma}, 0}
$$

3 We would like to thank N. Constable for pointing out this interpretation. 
$f$ in $(2.17)$ is given by

$$
f=\frac{1}{1+\widetilde{\lambda}}=\frac{1}{1+\lambda e^{x^{0}}} .
$$

From (2.11) and (2.17), we obtain the following decomposition of the boundary state:

$$
|B\rangle=f|D\rangle+\left|B_{0}^{\prime}\right\rangle=(f-1)|D\rangle+|N\rangle+\left|B_{1}^{\prime}\right\rangle
$$

Here, $|D\rangle$ and $|N\rangle$ are the usual Dirichlet and Neumann states

$$
|D\rangle=\exp \left(\sum_{n=1}^{\infty} \frac{1}{n} \alpha_{-n} \tilde{\alpha}_{-n}\right)|0\rangle, \quad|N\rangle=\exp \left(-\sum_{n=1}^{\infty} \frac{1}{n} \alpha_{-n} \tilde{\alpha}_{-n}\right)|0\rangle,
$$

and $\left|B_{k}^{\prime}\right\rangle(k=0,1)$ is the extra piece

$$
\left|B_{k}^{\prime}\right\rangle=-\sum_{N=k}^{\infty}(-\widetilde{\lambda})^{N} \sum_{\sigma, \tilde{\sigma}} \mathcal{N}^{\prime(\sigma, \tilde{\sigma})} \sum_{\ell(Y)>N} \chi_{Y}(\sigma) \chi_{Y}(\tilde{\sigma}) \exp \left(-\sum_{n=1}^{\infty} \frac{1}{n} \alpha_{-n} \tilde{\alpha}_{-n}\right)|\sigma, \tilde{\sigma}\rangle
$$

The exponentially growing terms found by Okuda and Sugimoto [13] are contained solely in $\left|B_{1}^{\prime}\right\rangle$. Note also that $|B\rangle$ reduces to the Neumann state $|N\rangle$ when $\lambda=0$, as expected.

From (2.16), one can see that the growing terms in $\left|B_{0}^{\prime}\right\rangle$ are coming from the failure of the completeness of $\chi_{Y}(\sigma)$ due to the constraint $\ell(Y) \leq N$. We expect that the constraint $\ell(N) \leq N$ becomes irrelevant in the large $N$ limit. In fact, it is well known that in the large $N$ limit the traces of unitary matrix behave as the free Gaussian variables [29]

$$
\lim _{N \rightarrow \infty} \int_{U(N)} d U F\left(\operatorname{Tr} U^{n}, \operatorname{Tr} U^{-n}\right)=\int \prod_{m=1}^{\infty} d \mu_{m} d \bar{\mu}_{m} e^{-\mu_{m} \bar{\mu}_{m}} F\left(\sqrt{n} \mu_{n}, \sqrt{n} \bar{\mu}_{n}\right) .
$$

Using this relation, it is easy to see that

$$
\lim _{N \rightarrow \infty} \int_{U(N)} d U\left|B_{U}\right\rangle=|D\rangle
$$

If we replace all the finite $N$ integral in (2.13) by the large $N$ value (2.24), we get

$$
|B\rangle \sim \sum_{N=0}^{\infty}(-\widetilde{\lambda})^{N}|D\rangle=f|D\rangle
$$

4 Since we compute the matrix integral by expanding the exponential (2.13), our large $N$ limit corresponds to the strong coupling phase of the Gross-Witten phase transition [30], in which the eigenvalue distribution is uniform on the unit circle. We will not discuss the interpretation of the weak coupling phase in the rolling tachyon boundary state. 
Therefore, the first term in the decomposition (2.20) can be thought of as the large $N$ result and the extra piece $\left|B_{0}^{\prime}\right\rangle$ corresponds to the finite $N$ effect.

Before concluding this subsection, let us comment on our treatment of the zero-mode $x^{0}$. In the boundary state $|B\rangle$, the boundary interaction $\int_{\partial \Sigma} e^{X^{0}}$ acts on the Neumann state at $\tau=0$ in the closed string channel. Since the conjugate momentum of $x^{0}$ does not appear in $X^{0}(\tau=0)$

$$
X^{0}(\tau=0)=x^{0}+p^{0} \tau+\text { oscillators }\left.\right|_{\tau=0}=x^{0}+\text { oscillators, }
$$

the zero-mode $x^{0}$ can be treated as if it is a c-number. For instance, $f$ in (2.19) should be understood as the eigenvalue in the coordinate basis [13]

$$
f \leftrightarrow \int d x^{0} f\left(x^{0}\right)\left|x^{0}\right\rangle
$$

It is interesting to compare this treatment of zero-mode with that in the spacelike Liouville theory. In the spacelike theory, it is sometimes useful to integrate out the zero-mode first and then integrate the non-zero modes [31]. On the other hand, in the timelike theory it turned out to be useful to integrate the zero-mode last and treat it as a part of the coupling [10,18].

\subsection{Relation to the $c=1$ Discrete States}

It was argued in [18 that the boundary state (1.3) written in terms of the discrete states and the matrix integral representation of the boundary state (2.13) are equivalent. This was checked up to the level 3 of oscillator number [18]. It is not so easy to see which terms in (2.13) correspond to the Virasoro Ishibashi state $|j, m, m\rangle\rangle$. However, in some cases we can identify the primary states $|j, m, m\rangle$ in the matrix representation of $|B\rangle(2.13)$. For instance, the level 0 term $f|0\rangle$ is obtained by summing over the primary states with $m=j$ [2,10,11]. We can also identify the primary state with $m=j-1$. The explicit form of $\left|B_{1}^{\prime}\right\rangle$ up to level 3 is

$$
\begin{aligned}
\left|B_{1}^{\prime}\right\rangle=\tilde{\lambda} & {\left[\frac{1}{2}\left(\alpha_{-2}-\sqrt{2} \alpha_{-1}^{2}\right)\left(\widetilde{\alpha}_{-2}-\sqrt{2} \widetilde{\alpha}_{-1}^{2}\right)\right.} \\
& \left.+\left(\frac{2}{3} \alpha_{-3}-\frac{1}{\sqrt{2}} \alpha_{-2} \alpha_{-1}-\frac{1}{3} \alpha_{-1}^{3}\right)\left(\frac{2}{3} \widetilde{\alpha}_{-3}-\frac{1}{\sqrt{2}} \widetilde{\alpha}_{-2} \widetilde{\alpha}_{-1}-\frac{1}{3} \widetilde{\alpha}_{-1}^{3}\right)+\cdots\right]|0\rangle \\
& -\widetilde{\lambda}^{2}\left[\left(\frac{\sqrt{2}}{3} \alpha_{-3}-\alpha_{-2} \alpha_{-1}+\frac{\sqrt{2}}{3} \alpha_{-1}^{3}\right)\left(\frac{\sqrt{2}}{3} \widetilde{\alpha}_{-3}-\widetilde{\alpha}_{-2} \widetilde{\alpha}_{-1}+\frac{\sqrt{2}}{3} \widetilde{\alpha}_{-1}^{3}\right)+\cdots\right]|0\rangle \\
& +\mathcal{O}\left(\widetilde{\lambda}^{3}\right) .
\end{aligned}
$$


On the other hand, the first three terms of the discrete states with $m=j-1$ are given by

$$
\begin{aligned}
|1,0,0\rangle_{\text {osc }} & =\alpha_{-1} \widetilde{\alpha}_{-1}|0\rangle \\
\left|\frac{3}{2}, \frac{1}{2}, \frac{1}{2}\right\rangle_{\text {osc }} & =\frac{1}{6}\left(\alpha_{-2}-\sqrt{2} \alpha_{-1}^{2}\right)\left(\widetilde{\alpha}_{-2}-\sqrt{2} \widetilde{\alpha}_{-1}^{2}\right)|0\rangle \\
|2,1,1\rangle_{\text {osc }} & =\frac{1}{4}\left(\frac{\sqrt{2}}{3} \alpha_{-3}-\alpha_{-2} \alpha_{-1}+\frac{\sqrt{2}}{3} \alpha_{-1}^{3}\right)\left(\frac{\sqrt{2}}{3} \widetilde{\alpha}_{-3}-\widetilde{\alpha}_{-2} \widetilde{\alpha}_{-1}+\frac{\sqrt{2}}{3} \widetilde{\alpha}_{-1}^{3}\right)|0\rangle
\end{aligned}
$$

where $|j, m, m\rangle_{o s c}$ denotes the oscillator part of discrete state. Comparing (2.28) and (2.29), we see that, for $n \leq 3$, the order $\widetilde{\lambda}^{n-1}$ term at level $n$ corresponds to the discrete state with $m=j-1$. We can show that this correspondence is true for general $n$, as follows. Up to the choice of phase, the discrete state operator $\mathcal{O}^{j, m=j-1}$ is given by

$$
\begin{aligned}
\mathcal{O}^{j, j-1} & =\frac{1}{\sqrt{2 j}} J^{-} \mathcal{O}^{j, j} \\
& =\frac{1}{\sqrt{2 j}} \oint \frac{d z}{2 \pi i} e^{-2 i X(z)} e^{2 i j X(0)} \\
& =\frac{1}{\sqrt{2 j}} \oint \frac{d z}{2 \pi i} z^{-2 j} \exp \left[2 i(j-1) X(0)-2 i \sum_{n=1}^{\infty} \frac{z^{n}}{n !} \partial^{n} X(0)\right] .
\end{aligned}
$$

Using the correspondence (2.6), the oscillator part of $\mathcal{O}^{j, j-1}$ is found to be

$$
|j, j-1\rangle_{\text {osc }}=\frac{1}{\sqrt{2 j}} \oint \frac{d z}{2 \pi i} z^{-2 j} \exp \left[-\sum_{n=1}^{\infty} \frac{\sqrt{2}}{n} \alpha_{-n} z^{n}\right]|0\rangle .
$$

In $\left|B_{0}^{\prime}\right\rangle(2.22)$, the order $\tilde{\lambda}^{n-1}$ term at level $n$ comes from the Young tableau $Y=\left(1^{n}\right)$, which has only one column. We denote the sum of those terms as $|b\rangle$. Using the relation

$$
\chi_{(1|\sigma|)}(\sigma)=(-1)^{|\sigma|-\ell(\sigma)},
$$

and recalling that $|\sigma, \tilde{\sigma}\rangle$ is at the level $|\sigma|,|b\rangle$ is written as

$$
\begin{aligned}
|b\rangle & =-\sum_{|\sigma|=|\widetilde{\sigma}|}(-\widetilde{\lambda})^{|\sigma|-1}(-1)^{|\sigma|-\ell(\sigma)+|\widetilde{\sigma}|-\ell(\tilde{\sigma})} \mathcal{N}^{\prime(\sigma, \widetilde{\sigma})}|\sigma, \tilde{\sigma}\rangle \\
& =\frac{1}{\widetilde{\lambda}} \int_{0}^{2 \pi} \frac{d \theta}{2 \pi} \exp \left[-\sum_{n=1}^{\infty} \frac{\sqrt{2}(-\tilde{\lambda})^{\frac{n}{2}}}{n}\left(\alpha_{-n} e^{i n \theta}+\widetilde{\alpha}_{-n} e^{-i n \theta}\right)\right]|0\rangle-\frac{1}{\widetilde{\lambda}}|0\rangle .
\end{aligned}
$$

Here, the $\theta$-integral was introduced to impose the level matching constraint $|\sigma|=|\tilde{\sigma}|$. From (2.31) and (2.33), we find

$$
|b\rangle=-\sum_{j=1, \frac{3}{2}, \cdots} 2 j(-\tilde{\lambda})^{2 j-2}|j, j-1, j-1\rangle_{o s c} .
$$


The coefficient $2 j$ in $|b\rangle$ is different from the coefficient $\left(\begin{array}{c}j+j-1 \\ 2(j-1)\end{array}\right)=2 j-1$ in (1.3). This implies that the state $|j, j-1, j-1\rangle$ with coefficient \pm 1 is contained in the leading term $f|D\rangle$ in the decomposition (2.20). This phenomenon was observed at level 2 in [13].

\subsection{Exponential Form of $|B\rangle$}

The matrix representation of boundary state (2.13) was obtained by expanding the boundary interaction. In this subsection, we will "re-exponentiate" the expression (2.13).

We first consider the exponential form of $|A\rangle$. In the eigenvalue basis, the matrix representation of $|A\rangle$ in 2.12 is rewritten as

$$
|\mathcal{A}\rangle=\sum_{N=0}^{\infty}(-\widetilde{\lambda})^{N} \frac{1}{N !} \int \prod_{k=1}^{N} \frac{d \theta_{k}}{2 \pi} \prod_{k<l}\left|e^{i \theta_{k}}-e^{i \theta_{l}}\right|^{2} \prod_{k=1}^{N} e^{2 i X^{(-)}\left(\theta_{k}\right)}|0\rangle
$$

where we defined $X^{( \pm)}$by

$$
\begin{aligned}
& X^{(+)}=\frac{i}{\sqrt{2}} \sum_{n>0} \frac{1}{n}\left[\alpha_{n} e^{-i n \theta}+\widetilde{\alpha}_{n} e^{i n \theta}\right], \\
& X^{(-)}=\frac{i}{\sqrt{2}} \sum_{n<0} \frac{1}{n}\left[\alpha_{n} e^{-i n \theta}+\widetilde{\alpha}_{n} e^{i n \theta}\right]=-\frac{i}{\sqrt{2}} \sum_{n>0} \frac{1}{n}\left[\alpha_{-n} e^{i n \theta}+\widetilde{\alpha}_{-n} e^{-i n \theta}\right] .
\end{aligned}
$$

The key observation is that the Vandermonde factor in (2.35) can be written as the commutator

$$
\begin{aligned}
{\left[i X^{(+)}\left(\theta_{k}\right), 2 i X^{(-)}\left(\theta_{l}\right)\right] } & =-\sum_{n=1}^{\infty} \frac{1}{n}\left[e^{-i n\left(\theta_{k}-\theta_{l}\right)}+e^{i n\left(\theta_{k}-\theta_{l}\right)}\right] \\
& =\log \left(1-e^{-i\left(\theta_{k}-\theta_{l}\right)}\right)+\log \left(1-e^{i\left(\theta_{k}-\theta_{l}\right)}\right) \\
& =\log \left|e^{i \theta_{k}}-e^{i \theta_{l}}\right|^{2} .
\end{aligned}
$$

Using the identity $e^{A} e^{B}=e^{B} e^{A} e^{[A, B]}$, we get the factor $\left|e^{i \theta_{k}}-e^{i \theta_{l}}\right|^{2}$ when exchanging the order of $e^{i X^{(+)}}$and $e^{2 i X^{(-)}}$:

$$
e^{i X^{(+)}\left(\theta_{k}\right)} e^{2 i X^{(-)}\left(\theta_{l}\right)}=e^{2 i X^{(-)}\left(\theta_{l}\right)} e^{i X^{(+)}\left(\theta_{k}\right)}\left|e^{i \theta_{k}}-e^{i \theta_{l}}\right|^{2}
$$

To write $|A\rangle$ in an exponential form, we introduce the operator

$$
\mathcal{V}=\int \frac{d \theta}{2 \pi} e^{2 i X^{(-)}(\theta)} e^{i X^{(+)}(\theta)}
$$


The $N^{\text {th }}$ power of $\mathcal{V}$ is written as

$$
\begin{aligned}
& \mathcal{V}^{N} \\
= & \int \frac{d \theta_{1}}{2 \pi} e^{2 i X^{(-)}\left(\theta_{1}\right)} e^{i X^{(+)}\left(\theta_{1}\right)} \int \frac{d \theta_{2}}{2 \pi} e^{2 i X^{(-)}\left(\theta_{2}\right)} e^{i X^{(+)}\left(\theta_{2}\right)} \cdots \int \frac{d \theta_{N}}{2 \pi} e^{2 i X^{(-)}\left(\theta_{N}\right)} e^{i X^{(+)}\left(\theta_{N}\right)} \\
= & \int \prod_{k=1}^{N} \frac{d \theta_{k}}{2 \pi} e^{2 i X^{(-)}\left(\theta_{1}\right)} e^{2 i X^{(-)}\left(\theta_{2}\right)} e^{i X^{(+)}\left(\theta_{1}\right)}\left|e^{i \theta_{1}}-e^{i \theta_{2}}\right|^{2} e^{i X^{(+)}\left(\theta_{2}\right)} \cdots e^{2 i X^{(-)}\left(\theta_{N}\right)} e^{i X^{(+)}\left(\theta_{N}\right)} \\
= & \int \prod_{k=1}^{N} \frac{d \theta_{k}}{2 \pi} \prod_{k<l}\left|e^{i \theta_{k}}-e^{i \theta_{l}}\right|^{2} \prod_{k=1}^{N} e^{2 i X^{(-)}\left(\theta_{k}\right)} \prod_{l=1}^{N} e^{i X^{(+)}\left(\theta_{l}\right)} \cdot
\end{aligned}
$$

In the second line, we changed the order of $e^{i X^{(+)}\left(\theta_{1}\right)}$ and $e^{2 i X^{(-)}\left(\theta_{2}\right)}$. We repeated this change of ordering until all the $e^{i X^{(+)}}$'s are on the right of $e^{2 i X^{(-)}}$'s. In this process we get the Vandermonde factor $\prod_{k<l}\left|e^{i \theta_{k}}-e^{i \theta_{l}}\right|^{2}$. Combining (2.35) and (2.40), and using the fact that $X^{(+)}(\theta)|0\rangle=0$, we can write $|A\rangle$ in an exponential form

$$
|A\rangle=\exp (-\tilde{\lambda} \mathcal{V})|0\rangle
$$

From (2.11), $|B\rangle$ is also written as

$$
|B\rangle=\exp \left(-\widetilde{\lambda} \mathcal{V}_{T}\right)|N\rangle
$$

where $\mathcal{V}_{T}$ is the tachyon operator given by

$$
\mathcal{V}_{T}=\exp \left(-\sum_{n=1}^{\infty} \frac{1}{n} \alpha_{-n} \widetilde{\alpha}_{-n}\right) \mathcal{V} \exp \left(\sum_{n=1}^{\infty} \frac{1}{n} \alpha_{-n} \widetilde{\alpha}_{-n}\right)=\int \frac{d \theta}{2 \pi} e^{2 i X^{(-)}} e^{i X^{(+)}-i X^{(-)}} .
$$

Note that the ordering in $\mathcal{V}_{T}$ is different from the ordinary normal ordering. If we define the "bare" coupling $\lambda_{0}$ as the coefficient in front of the normal ordered operator

$$
\lambda_{0} e^{i X^{(-)}} e^{i X^{(+)}}=\lambda e^{2 i X^{(-)}} e^{i X^{(+)}-i X^{(-)}},
$$

then $\lambda$ and $\lambda_{0}$ are related by

$$
\lambda=\lambda_{0} e^{\frac{1}{2}\left[i X^{(+)}, i X^{(-)}\right]}=\lambda_{0}\left|e^{i \theta}-e^{i \theta}\right|^{\frac{1}{2}} .
$$

To make the coupling $\lambda$ finite, we should introduce a cut-off and take the limit $\lambda_{0} \rightarrow \infty$. One might worry that this renormalization would lead to the breaking of the conformal invariance. In the next subsection, we directly check the conformal invariance of the matrix representation of $|B\rangle(2.13)$. 


\subsection{Conformal Invariance}

Let us check the conformal invariance of the boundary state $\left|B_{U(N)}\right\rangle$ with fixed $N$

$$
\left|B_{U(N)}\right\rangle \equiv \int_{U(N)} d U\left|B_{U}\right\rangle, \quad\left(\alpha_{n}+\widetilde{\alpha}_{-n}\right)\left|B_{U}\right\rangle=\gamma \operatorname{Tr} U^{n}\left|B_{U}\right\rangle
$$

Here we slightly generalized the condition (2.14) by replacing the coefficient $\sqrt{2}$ in front of $\operatorname{Tr} U^{n}$ by a constant $\gamma$. The level matching condition $\left(L_{0}-\widetilde{L}_{0}\right)\left|B_{U(N)}\right\rangle=0$ follows from the $U(1)$ symmetry $U \rightarrow e^{i \alpha} U$ of the matrix integral. To see the invariance under $L_{n}-\widetilde{L}_{-n}$, it is convenient to write the measure $d U$ as a contour integral around $z=0$

$$
\int_{U(N)} d U=\frac{1}{N !} \oint \prod_{k=1}^{N} \frac{d z_{k}}{2 \pi i z_{k}} \prod_{i<j}\left(z_{i}-z_{j}\right)\left(z_{i}^{-1}-z_{j}^{-1}\right) .
$$

Then the action of $L_{n}-\widetilde{L}_{-n}$ is represented by the change of integration variables

$$
z_{k} \rightarrow z_{k}+\varepsilon z_{k}^{n+1}
$$

By writing explicitly the variation of $\left|B_{U(N)}\right\rangle$ under (2.48), we can show that the condition $\left(L_{n}-\widetilde{L}_{-n}\right)\left|B_{U(N)}\right\rangle=0$ holds if and only if $\gamma^{2}=2$ 目. The two allowed values $\gamma= \pm \sqrt{2}$ correspond to the tachyon configurations $T=\lambda e^{ \pm X^{0}}$. After taking into account the open string creation and the closed string emission, the conformal invariance will be broken due to the divergence in both channels [16,19].

\subsection{The Leading Term $\left|B_{f}\right\rangle=f|D\rangle$}

The leading term of $|B\rangle$ in the decomposition (2.20) has the form

$$
\left|B_{f}\right\rangle \equiv f|D\rangle=\int d x^{0} f\left(x^{0}\right)\left|D_{x^{0}}\right\rangle .
$$

Therefore, $f\left(x^{0}\right)$ can be thought of as the distribution function of SD-branes localized on the real time axis. $\left|B_{f}\right\rangle$ roughly corresponds to the Lorentzian FZZ state (1.2)

$$
\left|B_{f}\right\rangle \simeq\left|B_{\mathrm{LFZZ}}\right\rangle
$$

5 In the literature of $\mathrm{YM}_{2}$, the correspondence between the oscillators and the trace of unitary matrix was usually written as $\alpha_{n}+\widetilde{\alpha}_{-n} \leftrightarrow \operatorname{Tr} U^{n}$, i.e., $\gamma=1$ [29, 32]. 
The wave function of the bulk primary operator $e^{i E X^{0}}$ seems to agree on both sides. However, the oscillator structure might be different between $\left|B_{f}\right\rangle$ and $\left|B_{\text {LFZZ }}\right\rangle$. In computing the wave function of $e^{i E X^{0}}$, i.e., the Fourier transform of $f\left(x^{0}\right)(2.19)$, we should be careful about the possible divergence coming from the region $x^{0} \sim-\infty$

$$
i \widehat{f}(E)=i \int_{-\infty}^{\infty} d x^{0} e^{i E x^{0}} f\left(x^{0}\right)=i \int_{-\infty}^{\infty} d x^{0} \frac{e^{i E x^{0}}}{1+\lambda e^{x^{0}}}
$$

In order to make this integral convergent, we should add an imaginary part to $E$ as $E \rightarrow E-i \varepsilon$, where $\varepsilon>0$. Then the integral becomes well-defined and the Fourier transform of $f$ is computed as

$$
i \widehat{f}(E)=\lambda^{-i E} \frac{\pi}{\sinh \pi(E-i \varepsilon)}
$$

By splitting $\widehat{f}(E)$ into the principal value part and the discontinuity part, $\widehat{f}(E)$ is written as

$$
i \widehat{f}(E)=\pi i \delta(E)+\lambda^{-i E} \mathbf{P}\left(\frac{\pi}{\sinh \pi E}\right)
$$

where $\mathbf{P}$ stands for the principal value

$$
\mathbf{P} F(E)=\lim _{\varepsilon \rightarrow 0} \frac{F(E+i \varepsilon)+F(E-i \varepsilon)}{2} .
$$

Since $1 / \sinh \pi E$ is an odd function of $E$, there is no singularity at $E=0$ in the principal value part

$$
\lim _{E \rightarrow 0} \mathbf{P}\left(\frac{\pi}{\sinh \pi E}\right)=0
$$

thus the singularity of $\widehat{f}(E)$ at $E=0$ is contained only in the delta-function term in (2.53). Since the open string tachyon stays near the top of the potential when $x^{0}<-\log \lambda$, the unstable brane can support the ends of open string for a very long time before it decays. This can be thought of as the origin of the singular term $\delta(E)$ in $\widehat{f}(E)$ [33].

The same form of the Fourier transform can be obtained by writing $f$ as

$$
\begin{aligned}
f\left(x^{0}\right) & =\frac{1}{2}-\frac{1}{2} \tanh \left(\frac{x^{0}+\log \lambda}{2}\right) \\
& =\frac{1}{2}-\sum_{n=-\infty}^{\infty} \frac{1}{x^{0}+\log \lambda-\pi i(2 n+1)} .
\end{aligned}
$$


The constant term $\frac{1}{2}$ gives to the delta-function in (2.53). Note that the pole $x^{0}=$ $-\log \lambda+\pi i(2 n+1)$ corresponds to the position of the imaginary branes discussed in [16, 17]. We can also write $f\left(x^{0}\right)$ as the resolvent of the matrix coordinate $\mathbf{X}^{0}$ of D-instantons

$$
f\left(x^{0}\right)=\frac{1}{2}-\operatorname{Tr} \frac{1}{x^{0}-\mathbf{X}^{0}}
$$

where $\mathbf{X}^{0}=\operatorname{diag}(-\log \lambda+\pi i(2 n+1))_{n \in \mathbf{Z}}$. Similarly, in the superstring case, the source of RR-field generated by the rolling tachyon can be written as a super-trace version of resolvent.

When computing the Fourier transform of $f$, we close the contour of $x^{0}$-integral in the upper (lower) half plane if $E>0(E<0)$, and pick up the poles of $f\left(x^{0}\right)$. Therefore, the in (out) state corresponds to the semi-infinite array of D-instantons sitting along the line $x^{0}=-\log \lambda$ on the upper (lower) half plane. In this way our decomposition (2.20) naturally explains the appearance of D-instanton array in the imaginary part of annulus amplitude [16]. The computation in [16] amounts to ignore the term $\left|B_{0}^{\prime}\right\rangle$. One may argue that $\left|B_{0}^{\prime}\right\rangle$ does not contribute to the imaginary part of annulus amplitude since all terms in $\left|B_{0}^{\prime}\right\rangle$ are "off-shell".

\section{Annulus Amplitude and Unitary Two-Matrix Integral}

In this section, we consider the annulus amplitude using the matrix representation of boundary state (2.13). Since the annulus has two boundary circles and the boundaries of worldsheet act as the space of eigenvalues of unitary matrix, the annulus amplitude is written as a unitary two-matrix integral.

Before writing the matrix representation of annulus, we emphasize that in general the Lorentzian annulus amplitude is not directly related to the Euclidean one. In the Euclidean case, the Ishibashi state is normalized as

$$
\left\langle\left\langle j, m, \widetilde{m}\left|q^{\frac{1}{2}\left(L_{0}+\widetilde{L}_{0}\right)-\frac{1}{24}}\right| j^{\prime}, m^{\prime}, \widetilde{m}^{\prime}\right\rangle\right\rangle=\delta_{j, j^{\prime}} \delta_{m, m^{\prime}} \delta_{\tilde{m}, \tilde{m}^{\prime}} \frac{q^{j^{2}}-q^{(j+1)^{2}}}{\eta(q)} .
$$

The conservation of momentum $\delta_{m, m^{\prime}}$ is due to the zero-mode integration. In the Lorentzian case, we cannot integrate over $x^{0}$ term by term, since each term has the form $e^{m x^{0}}$ which diverges at late times. As we mentioned in section 2.1, in the timelike Liouville theory it is useful to integrate out the non-zero modes first. In this paper, we will try to 
define the annulus amplitude by first contracting the oscillator part and then performing a resummation to make the integrand Fourier transformable, if possible.

So we consider the annulus amplitude without the zero-mode integration:

$$
Z\left(\widetilde{\lambda}_{1}, \widetilde{\lambda}_{2}\right)=\left\langle B\left(\widetilde{\lambda}_{1}\right)\left|q^{\frac{1}{2}(N+\tilde{N})-\frac{1}{24}}\right| B\left(\widetilde{\lambda}_{2}\right)\right\rangle .
$$

As before, we treat the zero-mode as a part of the coupling $\tilde{\lambda}=\lambda e^{x^{0}}$. To compute $Z\left(\widetilde{\lambda}_{1}, \widetilde{\lambda}_{2}\right)$, we can make use of the formula of oscillator contraction

$$
\begin{aligned}
\left\langle 0\left|\exp \left[a_{i} T_{i j} b_{j}+\lambda_{i}^{a} a_{i}+\lambda_{i}^{b} b_{i}\right] \exp \left[a_{i}^{\dagger} S_{i j} b_{j}^{\dagger}+\mu_{i}^{a} a_{i}^{\dagger}+\mu_{i}^{b} b_{i}^{\dagger}\right]\right| 0\right\rangle \\
=\frac{1}{\operatorname{det}_{i j}(1-T S)} \exp \left[\lambda_{i}^{a}\left(\frac{1}{1-T S}\right)_{i j} \mu_{j}^{a}+\mu_{i}^{b}\left(\frac{1}{1-T S}\right)_{i j} \lambda_{j}^{b}\right. \\
\left.+\mu_{i}^{b}\left(\frac{T}{1-T S}\right)_{i j} \mu_{j}^{a}+\lambda_{i}^{a}\left(\frac{S}{1-T S}\right)_{i j} \lambda_{j}^{b}\right],
\end{aligned}
$$

where $\lambda_{i}^{a, b}, \mu_{i}^{a, b}, S_{i j}$ and $T_{i j}$ are c-number parameters, and $a_{i}, b_{i}$ are normalized oscillators

$$
\left[a_{i}, a_{j}^{\dagger}\right]=\left[b_{i}, b_{j}^{\dagger}\right]=\delta_{i j}, \quad\left[a_{i}, b_{j}^{\dagger}\right]=0 .
$$

This formula assumes that $S_{i j}$ and $T_{i j}$ are symmetric matrices and commute with each other. We use this formula by identifying $a_{i} \leftrightarrow \alpha_{n} / \sqrt{n}$ and $b_{i} \leftrightarrow \widetilde{\alpha}_{n} / \sqrt{n}$. Then $Z\left(\widetilde{\lambda}_{1}, \widetilde{\lambda}_{2}\right)$ is evaluated as

$$
Z\left(\widetilde{\lambda}_{1}, \widetilde{\lambda}_{2}\right)=\frac{1}{\eta(q)} \sum_{N, M=0}^{\infty}\left(-\widetilde{\lambda}_{1}\right)^{N}\left(-\widetilde{\lambda}_{2}\right)^{M} Z_{N, M},
$$

where $Z_{N, M}$ is given by

$$
\begin{array}{r}
Z_{N, M}=\int_{U(N) \times U(M)} d U d V \exp \left[\sum _ { n = 1 } ^ { \infty } \frac { 2 } { n ( 1 - q ^ { n } ) } \left(q^{\frac{n}{2}} \operatorname{Tr} U^{n} \operatorname{Tr} V^{-n}+q^{\frac{n}{2}} \operatorname{Tr} U^{-n} \operatorname{Tr} V^{n}\right.\right. \\
\left.\left.-q^{n} \operatorname{Tr} U^{n} \operatorname{Tr} U^{-n}-q^{n} \operatorname{Tr} V^{n} \operatorname{Tr} V^{-n}\right)\right] .
\end{array}
$$

Namely, the annulus amplitude is written as the grand canonical partition function of the unitary two-matrix model with the double trace interactions.

$Z_{N, M}$ can be also written in a determinant form by expanding the factor $\left(1-q^{n}\right)^{-1}=$ $\sum_{m \geq 0} q^{n m}$, and summing over $n$ using $\sum_{n>0} x^{n} / n=-\log (1-x)$. In this way, we get

$$
\begin{aligned}
Z_{N, M}= & \int_{U(N) \times U(M)} d U d V \exp \left[\sum_{n, m=1}^{\infty} \frac{2}{n} q^{n\left(m-\frac{1}{2}\right)}\left\{\operatorname{Tr}\left(U \otimes V^{-1}\right)^{n}+\operatorname{Tr}\left(U^{-1} \otimes V\right)^{n}\right\}\right. \\
& \left.-\frac{2}{n} q^{n m}\left\{\operatorname{Tr}\left(U \otimes U^{-1}\right)^{n}+\operatorname{Tr}\left(V \otimes V^{-1}\right)^{n}\right\}\right] \\
= & \int_{U(N) \times U(M)} d U d V \prod_{m=1}^{\infty} \frac{\operatorname{det}\left(1-q^{m} U \otimes U^{-1}\right)^{2} \operatorname{det}\left(1-q^{m} V \otimes V^{-1}\right)^{2}}{\operatorname{det}\left(1-q^{m-\frac{1}{2}} U \otimes V^{-1}\right)^{2} \operatorname{det}\left(1-q^{m-\frac{1}{2}} U^{-1} \otimes V\right)^{2}}
\end{aligned}
$$


Here we have also used the relation $\operatorname{Tr} A \operatorname{Tr} B=\operatorname{Tr}(A \otimes B)$.

The large $N, M$ limit of $Z_{N, M}$ is obtained by the Gaussian integral (2.23)

$$
\lim _{N, M \rightarrow \infty} Z_{N, M}=\prod_{n=1}^{\infty} \operatorname{det}\left[\left(\begin{array}{ll}
1 & 0 \\
0 & 1
\end{array}\right)-\left(\begin{array}{cc}
\frac{-2 q^{n}}{1-q^{n}} & \frac{2 q^{\frac{n}{2}}}{1-q^{n}} \\
\frac{2 q^{\frac{n}{2}}}{1-q^{n}} & \frac{-2 q^{n}}{1-q^{n}}
\end{array}\right)\right]^{-1}=1
$$

Therefore, if we replace all the finite $N$ integrals in (3.5) by the large $N$ value (3.8), $Z\left(\widetilde{\lambda}_{1}, \widetilde{\lambda}_{2}\right)$ reduces to the annulus amplitude between the leading term $\left|B_{f}\right\rangle$

$Z\left(\widetilde{\lambda}_{1}, \widetilde{\lambda}_{2}\right) \sim \frac{1}{\eta(q)} \sum_{N, M=0}^{\infty}\left(-\widetilde{\lambda}_{1}\right)^{N}\left(-\widetilde{\lambda}_{2}\right)^{M}=\frac{1}{\eta(q)} \frac{1}{\left(1+\widetilde{\lambda}_{1}\right)\left(1+\widetilde{\lambda}_{2}\right)}=\left\langle B_{f_{1}}\left|q^{\frac{1}{2}(N+\widetilde{N})-\frac{1}{24}}\right| B_{f_{2}}\right\rangle$

From (3.7) and (3.8), we can see that the large $N$ limit of $Z_{N, M}$ is equivalent to the limit $\lim _{q \rightarrow 0} Z_{N, M}=1$. Namely, the large $N$ limit amounts to neglect the contribution from the exponentially growing timelike oscillators found in [13]. Note that the partition function of $b c$-ghosts $\eta(q)^{2}$ does not cancel the $q$-dependence in $Z_{N, M}$.

The amplitude with zero-mode integrated is given by

$$
\left\langle B\left|q^{\frac{1}{2}\left(L_{0}+\widetilde{L}_{0}\right)-\frac{1}{24}}\right| B\right\rangle=\int_{-\infty}^{\infty} \frac{d E}{2 \pi} d x^{0} d y^{0} q^{-\frac{1}{4} E^{2}} e^{-i E\left(x^{0}-y^{0}\right)} Z\left(\lambda e^{x^{0}}, \lambda e^{y^{0}}\right) .
$$

Without some appropriate prescription, all three integrals $\int d E, \int d x^{0}$ and $\int d y^{0}$ are illdefined because the integrand is divergent at infinity. Our proposal is to perform a resummation in (3.5) in order to make the integral over the zero-modes $x^{0}$ and $y^{0}$ convergent. If this resummation is possible and the Fourier transform of $Z\left(\lambda e^{x^{0}}, \lambda e^{y^{0}}\right)$ becomes welldefined, the remaining integral $\int d E$ can be treated in the same way as in [19].

\subsection{Some Examples of Matrix Integral}

In this subsection, we compute the integral $Z_{N, M}$ (3.6) for some cases. Since $Z_{N, M}$ is symmetric in $N$ and $M, Z_{N, M}=Z_{M, N}$, it is sufficient to consider the cases $N \geq M$. Let us first compute the integral $Z_{N, M}$ with $M=0$,

$$
\begin{aligned}
Z_{N, 0} & =\int_{U(N)} d U \exp \left(-\sum_{n=1}^{\infty} \frac{2 q^{n}}{n\left(1-q^{n}\right)} \operatorname{Tr} U^{n} \operatorname{Tr} U^{-n}\right) \\
& =\int_{U(N)} d U \prod_{n=1}^{\infty} \operatorname{det}\left(1-q^{n} U \otimes U^{-1}\right)^{2} .
\end{aligned}
$$


This integral also appears in the amplitude $\left\langle N\left|q^{\frac{1}{2}(N+\widetilde{N})-\frac{1}{24}}\right| B\right\rangle$ between the Neumann state and the rolling tachyon state. Since the $U(1)$ part of $U(N)$ integral is decoupled in (3.11), $\int_{U(N)}$ can be replaced by $\int_{S U(N)}$. This integral is computable thanks to the fact that the square root of the integrand is proportional to the Weyl denominator of affine $A_{N-1}$. Using the Weyl denominator formula of $\widehat{A}_{N-1}$ [35], we can expand the square root of integrand in terms of the $S U(N)$ characters $\chi_{\lambda}(U)$

$$
\begin{aligned}
\prod_{m=1}^{\infty} \operatorname{det}\left(1-q^{m} U \otimes U^{-1}\right) & =\prod_{n=1}^{\infty}\left(1-q^{n}\right) \sum_{\alpha \in \Lambda_{R}} \chi_{N \alpha}(U) q^{\frac{1}{2 N} C_{2}(N \alpha)} \\
& =\prod_{n=1}^{\infty}\left(1-q^{n}\right) \sum_{\alpha \in \Lambda_{R}} \chi_{N \alpha}\left(U^{-1}\right) q^{\frac{1}{2 N} C_{2}(N \alpha)}
\end{aligned}
$$

where $\Lambda_{R}$ is the root lattice of $S U(N)$, and $C_{2}(\lambda)=(\lambda, \lambda+2 \rho)$ is the second Casimir with $\rho$ being the half of the sum of positive roots. $\frac{1}{6}$ Using the orthogonality of the characters, $Z_{N, 0}$ becomes

$$
Z_{N, 0}=\prod_{n=1}^{\infty}\left(1-q^{n}\right)^{2} \sum_{\alpha \in \Lambda_{R}} q^{\frac{1}{N} C_{2}(N \alpha)} .
$$

The summation over $\Lambda_{R}$ can be performed by substituting the following "ground state" configuration $U_{0}$ into (3.12) [36,37]

$$
U_{0}=\operatorname{diag}\left(\omega^{n_{F}}, \omega^{n_{F}-1}, \cdots, \omega^{-n_{F}}\right) ; \quad \omega=e^{2 \pi i / N}, \quad n_{F}=\frac{N-1}{2} .
$$

Note that the eigenvalues of $U_{0}$ are distributed on the unit circle in a $\mathbf{Z}_{N}$-symmetric way. The special property of $U_{0}$ is that $\chi_{N \alpha}\left(U_{0}\right)=1$ for all $\alpha \in \Lambda_{R}$. Therefore, after replacing $q$ by $q^{2}$ in (3.12), the summation in (3.13) is written as 8

$$
\sum_{\alpha \in \Lambda_{R}} q^{\frac{1}{N} C_{2}(N \alpha)}=\prod_{m=1}^{\infty} \frac{\operatorname{det}\left(1-q^{2 m} U_{0} \otimes U_{0}^{-1}\right)}{1-q^{2 m}}=\prod_{m=1}^{\infty} \frac{\left(1-q^{2 N m}\right)^{N}}{1-q^{2 m}} .
$$

6 Similar integral has appeared in the inner product of the wave-functions of Chern-Simons theory on $T^{2} \times I$ 34

7 Note that eq. (3.12) is reminiscent of the disk amplitude of $\mathrm{YM}_{2}$ with finite area. Here the closed string proper time plays the role of the area. However this analogy is not exact, since in (3.12) only the sublattice $N \Lambda_{R}$ of the weight lattice $\Lambda_{w}$ contributes to the summation.

8 This is a version of the Macdonald identity [36]. In the combinatorial language, this sum is related to the generating function of the " $N$-core" of partitions [38]. 
One may think of $U_{0}$ as a kind of "master field". In the large $N$ limit, the eigenvalue distribution of $U_{0}$ approaches the uniform distribution on the unit circle. Finally, $Z_{N, 0}$ is found to be

$$
Z_{N, 0}=\prod_{m=1}^{\infty} \frac{\left(1-q^{m}\right)\left(1-q^{2 N m}\right)^{N}}{1+q^{m}}
$$

In the general case $N, M \neq 0$, the square root of the integrand of $Z_{N, M}$ is proportional to the Weyl denominator of affine superalgebra $\widehat{A}(N-1, M-1)$ 39 8 . Although the denominator identity of $\widehat{A}(N-1, M-1)$ is known unless $N=M$, it is not in a form of the character expansion, which was useful for the $U(N)$ case (3.12). Because of this, so far we have not succeeded in computing the integral $Z_{N, M}$ for general $N, M$. Below, we compute a few terms of $Z_{N, M}$.

For example, using the $\widehat{A}(1,0)$ denominator identity [39,40

$$
\begin{aligned}
\widehat{R}(x, y) & =\prod_{n=1}^{\infty} \frac{\left(1-q^{n}\right)^{2}\left(1-q^{n} x y\right)\left(1-q^{n-1} x^{-1} y^{-1}\right)}{\left(1-q^{n-\frac{1}{2}} x\right)\left(1-q^{n-\frac{1}{2}} x^{-1}\right)\left(1-q^{n-\frac{1}{2}} y\right)\left(1-q^{n-\frac{1}{2}} y^{-1}\right)} \\
& =\left(\sum_{m, n \geq 0}-\sum_{m, n<0}\right) x^{m} y^{n} q^{m n+\frac{1}{2}(m+n)},
\end{aligned}
$$

$Z_{2,1}$ is evaluated as

$$
\begin{aligned}
Z_{2,1} & =\prod_{m=1}^{\infty}\left(1-q^{m}\right)^{2} \frac{1}{2 !} \int_{0}^{2 \pi} \frac{d \theta_{1} d \theta_{2} d \phi}{(2 \pi)^{3}} \widehat{R}\left(e^{i \theta_{1}-i \phi}, e^{-i \theta_{2}+i \phi}\right) \widehat{R}\left(e^{-i \theta_{1}+i \phi}, e^{i \theta_{2}-i \phi}\right) \\
& =\prod_{m=1}^{\infty}\left(1-q^{m}\right)^{2} \sum_{n=0}^{\infty} \frac{q^{n}}{1-q^{2 n+1}} .
\end{aligned}
$$

We can also compute $Z_{1,1}$ defined by

$$
Z_{1,1}=\int_{0}^{2 \pi} \frac{d \theta}{2 \pi} \int_{0}^{2 \pi} \frac{d \phi}{2 \pi} \prod_{m=1}^{\infty} \frac{\left(1-q^{m}\right)^{4}}{\left(1-q^{m-\frac{1}{2}} e^{i \theta-i \phi}\right)^{2}\left(1-q^{m-\frac{1}{2}} e^{-i \theta+i \phi}\right)^{2}}
$$

This integral can be evaluated by using the identity,

$$
\prod_{n=1}^{\infty} \frac{\left(1-q^{n}\right)^{4}}{\left(1-q^{n-\frac{1}{2}} z\right)^{2}\left(1-q^{n-\frac{1}{2}} z^{-1}\right)^{2}}=\sum_{m, n \geq 0}^{\infty}(m+n+1) z^{m-n} q^{m n+\frac{1}{2}(m+n)}
$$

9 In [39], the Weyl denominator which is relevant for our integral is denoted as $\widehat{R}_{T}$. 
which is obtained as a limit of (3.17), and we get

$$
Z_{1,1}=\sum_{n=0}^{\infty}(2 n+1) q^{n(n+1)} .
$$

Since we do not have a closed form of $Z_{N, M}$ for general $N, M$, 10 we cannot discuss whether or not the resummation in (3.5) is possible. In the next section, we consider an example of annulus amplitude in which we can perform a resummation explicitly.

\section{Annulus Amplitude between $\left|B_{f}\right\rangle$ and $|B\rangle$}

In this section, we show that if we replace one of the boundary states in (3.2) by its leading term the contribution from the exponentially growing timelike oscillators 13 can be resummed. Namely, we consider the following amplitude without the zero-mode integral:

$$
\left\langle B_{f}\left|q^{\frac{1}{2}(N+\widetilde{N})-\frac{1}{24}}\right| B\right\rangle=f\left\langle D\left|q^{\frac{1}{2}(N+\widetilde{N})-\frac{1}{24}}\right| B\right\rangle .
$$

This can be thought of as a part of the amplitude between two $|B\rangle$ 's.

\subsection{Matrix Integral}

Since the zero-mode dependence of $\left\langle B_{f}\right|$ is factorized as $f\langle D|$, we focus on the oscillator contraction between $\langle D|$ and $|B\rangle$ :

$$
Z_{D B}=\left\langle D\left|q^{\frac{1}{2}(N+\widetilde{N})-\frac{1}{24}}\right| B\right\rangle=q^{-\frac{1}{24}} \prod_{n=1}^{\infty}\left(1+q^{n}\right)^{-1} \sum_{N=0}^{\infty}(-\widetilde{\lambda})^{N} F_{N}
$$

where $F_{N}$ is given by

$$
\begin{aligned}
F_{N} & =\int_{U(N)} d U \exp \left(\sum_{n=1}^{\infty} \frac{2 q^{n}}{n\left(1+q^{n}\right)} \operatorname{Tr} U^{n} \operatorname{Tr} U^{-n}\right) \\
& =\int_{U(N)} d U \prod_{n=1}^{\infty} \frac{\operatorname{det}\left(1-q^{2 n} U \otimes U^{-1}\right)^{2}}{\operatorname{det}\left(1-q^{2 n-1} U \otimes U^{-1}\right)^{2}} .
\end{aligned}
$$

10 We have computed first few terms of $Z_{3,1}$ and $Z_{2,2}$ by using Mathematica:

$$
\begin{aligned}
& Z_{3,1}=1-3 q^{2}-q^{4}+6 q^{6}+7 q^{8}-10 q^{10}-9 q^{12}-q^{14}+\mathcal{O}\left(q^{17}\right), \\
& Z_{2,2}=1+8 q^{3}+27 q^{8}+64 q^{15}+\mathcal{O}\left(q^{17}\right) .
\end{aligned}
$$


$F_{0}$ and $F_{1}$ are easily found to be

$$
F_{0}=1, \quad F_{1}=\prod_{n=1}^{\infty} \frac{\left(1-q^{2 n}\right)^{2}}{\left(1-q^{2 n-1}\right)^{2}} .
$$

As before, the square root of the integrand of $F_{N}$ is related to the Weyl denominator of some affine superalgebra. In this case, the relevant algebra is the so-called $Q(N-1)$ superalgebra [39]. For example, using the denominator identity of $Q(1)$ affine superalgebra

$$
\widehat{R}(x)=\prod_{n=1}^{\infty} \frac{\left(1-q^{2 n}\right)^{2}\left(1-q^{2 n-2} x\right)\left(1-q^{2 n} x^{-1}\right)}{\left(1-q^{2 n-1}\right)^{2}\left(1-q^{2 n-1} x\right)\left(1-q^{2 n-1} x^{-1}\right)}=\sum_{k=-\infty}^{\infty} \frac{q^{k}}{1-q^{2 k+1} x},
$$

$F_{2}$ is evaluated as

$$
F_{2}=\frac{1}{2 !} \int_{0}^{2 \pi} \frac{d \theta_{1} d \theta_{2}}{(2 \pi)^{2}} \widehat{R}\left(e^{i \theta_{1}-i \theta_{2}}\right) \widehat{R}\left(e^{-i \theta_{1}+i \theta_{2}}\right)=\sum_{k=0}^{\infty} \frac{q^{2 k}}{\left(1-q^{2 k+1}\right)^{2}} .
$$

Similarly, $F_{3}$ is obtained by using the denominator identity of $Q(2)$ [39,41]

$$
F_{3}=\prod_{n=1}^{\infty} \frac{\left(1-q^{2 n}\right)^{2}}{\left(1-q^{2 n-1}\right)^{2}} \sum_{k=0}^{\infty} \frac{q^{2 k}}{\left(1-q^{2 k+2}\right)^{2}} .
$$

For $Q(N-1)$ affine superalgebra with $N>3$, there is only a conjectured denominator formula which has not been proved yet [39]. Instead of computing the matrix integral directly, in the next subsection we consider a different approach.

\subsection{Computation of $Z_{D B}$}

$Z_{D B}$ can be obtained by making use of the knowledge of Euclidean annulus amplitude. As we emphasized in section 3, in general the Lorentzian annulus amplitude is not directly related to the Euclidean one. In particular, we need the matrix element without the zeromode integration

$$
{ }_{o s c}\left\langle\left\langle j, m, \widetilde{m}\left|q^{\frac{1}{2}(N+\widetilde{N})-\frac{1}{24}}\right| j^{\prime}, m^{\prime}, \widetilde{m}^{\prime}\right\rangle\right\rangle_{o s c} .
$$

In general, this amplitude is not diagonal in $m .11$ However, $Z_{D B}$ is related to the Euclidean amplitude as follows. The amplitude $Z_{D B}$ without zero-mode integral is written as

$$
Z_{D B}=\left\langle D\left|q^{\frac{1}{2}(N+\widetilde{N})-\frac{1}{24}}\right| B\right\rangle=\left\langle D \mid q^{\frac{1}{2}(N+\widetilde{N})-\frac{1}{24}}\left[\sum_{j, m \geq 0}\left(\begin{array}{c}
j+m \\
2 m
\end{array}\right)(-1)^{m} \widetilde{\lambda}^{2 m}|j, m, m\rangle\right\rangle_{\text {osc }}\right] .
$$

11 From (2.15), the off-diagonal metrix elements (4.8) correspond to the integrals $Z_{N, M}$ with $N \neq M$. Especially, these terms are necessary to have the factorized form $f_{1} f_{2}$ in (3.9). 
The important fact here is that the matrix element

$$
\left.\left\langle D\left|q^{\frac{1}{2}(N+\widetilde{N})-\frac{1}{24}}\right| j, m, m\right\rangle\right\rangle_{o s c}
$$

appearing in (4.9) is equal to the Euclidean amplitude with zero-mode integrated

$$
\left.q^{-m^{2}}\left\langle D ; m, m\left|q^{\frac{1}{2}\left(L_{0}+\widetilde{L}_{0}\right)-\frac{1}{24}}\right| j, m, m\right\rangle\right\rangle
$$

where we defined

$$
\langle D ; m, m|=\left\langle\alpha_{0}=\widetilde{\alpha}_{0}=\sqrt{2} m\right| \exp \left(\sum_{n=1}^{\infty} \frac{1}{n} \alpha_{n} \widetilde{\alpha}_{n}\right)
$$

The factor $q^{-m^{2}}$ in (4.11) cancels the weight from the zero-mode $\frac{1}{2} \alpha_{0}^{2}=m^{2}$, thus (4.11) is equal to the oscillator contraction (4.10). In other words, we add the Euclidean zero-mode by hand and subtract its effect by the factor $q^{-m^{2}}$. Again, we emphasize that we use the Euclidean amplitude (4.11) just as a technical tool to compute the Lorentzian amplitude. Now $Z_{D B}$ is written as

$$
\begin{aligned}
Z_{D B} & \left.=\sum_{j, m \geq 0}\left(\begin{array}{c}
j+m \\
2 m
\end{array}\right)(-1)^{m} \widetilde{\lambda}^{2 m} q^{-m^{2}}\left\langle D ; m, m\left|q^{\frac{1}{2}\left(L_{0}+\widetilde{L}_{0}\right)-\frac{1}{24}}\right| j, m, m\right\rangle\right\rangle \\
& =\sum_{m^{\prime} \in \frac{1}{2} \mathbf{Z}}\left\langle D ; m^{\prime}, m^{\prime} \mid q^{\frac{1}{2}\left(L_{0}+\widetilde{L}_{0}\right)-\frac{1}{24}}\left[\sum_{j, m \geq 0} q^{-m^{2}}\left(\begin{array}{c}
j+m \\
2 m
\end{array}\right)(-1)^{m} \widetilde{\lambda}^{2 m}|j, m, m\rangle\right\rangle\right] \\
& =\left\langle D_{S U(2)} \mid q^{\frac{1}{2}\left(L_{0}+\widetilde{L}_{0}\right)-\frac{1}{24}}\left[\sum_{j, m \geq 0} q^{-m^{2}}\left(\begin{array}{c}
j+m \\
2 m
\end{array}\right)(-1)^{m} \widetilde{\lambda}^{2 m}|j, m, m\rangle\right\rangle\right]
\end{aligned}
$$

where $\left\langle D_{S U(2)}\right|$ is given by 24

$$
\left\langle D_{S U(2)}\right|=\sum_{m^{\prime} \in \frac{1}{2} \mathbf{Z}}\left\langle D ; m^{\prime}, m^{\prime}\right|=\sum_{j, m}\left\langle\langle j, m, m|(-1)^{j} .\right.
$$

In the second line of (4.13), we used the momentum conservation in the Euclidean amplitude. Using (3.1), we finally find

$$
Z_{D B}=\frac{1}{\eta(q)} \sum_{j, m \geq 0}\left(\begin{array}{c}
j+m \\
2 m
\end{array}\right)(-1)^{j+m} \widetilde{\lambda}^{2 m} q^{-m^{2}}\left(q^{j^{2}}-q^{(j+1)^{2}}\right) .
$$


Note that we cannot use this trick to compute $\left\langle B\left|q^{\frac{1}{2}(N+\widetilde{N})-\frac{1}{24}}\right| B\right\rangle$ since in this case we need to know the general "off-diagonal" amplitude (4.8).12 One can easily check that the first two terms of (4.15) in the $\widetilde{\lambda}$-expansion agree with the matrix computation (4.4). The agreement at the order $\widetilde{\lambda}^{2}$ and $\widetilde{\lambda}^{3}$ requires the following identities:

$$
\begin{aligned}
F_{2} & =\sum_{k=0}^{\infty} \frac{q^{2 k}}{\left(1-q^{2 k+1}\right)^{2}}=\prod_{n=1}^{\infty} \frac{1+q^{n}}{1-q^{n}} \sum_{k=0}^{\infty}(-1)^{k}(k+1)^{2} q^{k(k+2)}, \\
F_{3} & =\prod_{n=1}^{\infty} \frac{\left(1-q^{2 n}\right)^{2}}{\left(1-q^{2 n-1}\right)^{2}} \sum_{k=0}^{\infty} \frac{q^{2 k}}{\left(1-q^{2 k+2}\right)^{2}} \\
& =\prod_{n=1}^{\infty} \frac{1+q^{n}}{1-q^{n}} \sum_{k=0}^{\infty} \frac{(-1)^{k}}{6}(k+1)(k+2)(2 k+3) q^{k(k+3)} .
\end{aligned}
$$

Eqs. (4.17a, b) can be proved by taking a derivative of the Jacobi's triple product identity (see appendix A for details). We believe that the two expressions (4.2) and (4.15) are equivalent for all orders in $\widetilde{\lambda}$.

$Z_{D B}$ can also be written in the form of $S U(2)_{k=1}$ character as in [20]

$$
Z_{D B}=\frac{q^{-\frac{1}{4} \partial_{0}^{2}}}{\eta(q)}\left[1+2 \sum_{n=1}^{\infty}(-1)^{n} q^{n^{2}} \cos 2 n \alpha-2 \sum_{n=0}^{\infty}(-1)^{n} q^{\left(n+\frac{1}{2}\right)^{2}} \sin (2 n+1) \alpha\right]
$$

where we defined

$$
\alpha=\arcsin \left(\frac{1}{2} \tilde{\lambda}\right)
$$

One might think that $\widetilde{\lambda}=2$ is a critical value above which $\alpha$ aquires an imaginary part and the amplitude becomes divergent as $\tilde{\lambda} \rightarrow \infty$. But this is merely a rephrasing of the fact that at a fixed level the coupling to the timelike oscillators grows exponentially. However, there is a room to perform a resummation over terms at different levels. In the next subsection, we will consider this possibility.

12 The diagonal integral $Z_{N, N}(3.6)$ can be obtained by using this trick:

$$
Z_{N, N}=\sum_{k=0}^{\infty}\left[\left(\begin{array}{c}
N+k \\
N
\end{array}\right)^{2}-\left(\begin{array}{c}
N-1+k \\
N
\end{array}\right)^{2}\right] q^{k(k+N)}
$$

For $N=1,2$, eq.(4.16) reproduces the direct matrix computation (3.21) and (3.22). 


\subsection{Resummation of Timelike Oscillators}

To perform a resummation in (4.15), we write $j=m+k(k=0,1, \cdots)$ and sum over $m$ with fixed $k$, and then sum over $k$. The amplitude with fixed $k=j-m$ is

$$
\begin{aligned}
Z_{D B}^{(k)} & =\frac{1}{\eta(q)} \sum_{m=0, \frac{1}{2}, 1, \cdots}^{\infty}(-1)^{2 m+k}\left(\begin{array}{c}
2 m+k \\
2 m
\end{array}\right)\left[\left(\widetilde{\lambda} q^{k}\right)^{2 m} q^{k^{2}}-\left(\widetilde{\lambda} q^{k+1}\right)^{2 m} q^{(k+1)^{2}}\right] \\
& =\frac{(-1)^{k}}{\eta(q)} \sum_{n=0}^{\infty}\left(\begin{array}{c}
n+k \\
n
\end{array}\right)\left[\left(-\widetilde{\lambda} q^{k}\right)^{n} q^{k^{2}}-\left(-\widetilde{\lambda} q^{k+1}\right)^{n} q^{(k+1)^{2}}\right] \\
& =\frac{(-1)^{k}}{\eta(q)}\left[\frac{q^{k^{2}}}{\left(1+\widetilde{\lambda} q^{k}\right)^{k+1}}-\frac{q^{(k+1)^{2}}}{\left(1+\widetilde{\lambda} q^{k+1}\right)^{k+1}}\right] .
\end{aligned}
$$

Therefore we get

$$
\begin{aligned}
Z_{D B} & =\frac{1}{\eta(q)} \sum_{k=0}^{\infty}(-1)^{k}\left[\frac{q^{k^{2}}}{\left(1+\widetilde{\lambda} q^{k}\right)^{k+1}}-\frac{q^{(k+1)^{2}}}{\left(1+\widetilde{\lambda} q^{k+1}\right)^{k+1}}\right] \\
& =\frac{1}{\eta(q)}\left[\frac{1}{1+\widetilde{\lambda}}+\sum_{k=1}^{\infty}(-1)^{k} q^{k^{2}} \frac{2+\widetilde{\lambda} q^{k}}{\left(1+\widetilde{\lambda} q^{k}\right)^{k+1}}\right]
\end{aligned}
$$

This expression is finite in the limit $\tilde{\lambda} \rightarrow \infty$. The first term in the second line is $f\left\langle D\left|q^{\frac{1}{2}(N+\widetilde{N})-\frac{1}{24}}\right| D\right\rangle$ which comes from the $k=0$ term, i.e., the $m=j$ term studied in the original work by Sen. The second term is $\left\langle D\left|q^{\frac{1}{2}(N+\widetilde{N})-\frac{1}{24}}\right| B_{0}^{\prime}\right\rangle$, which includes the contribution of the exponentially growing terms found in [13]. Namely, after performing a resummation over different levels, the contribution from $\left|B_{0}^{\prime}\right\rangle$ becomes finite!

Now we can perform the Fourier transformation of $Z_{D B}$ safely. Note that (4.21) exhibits an interesting interplay between the zero-mode $x^{0}$ and the closed string proper time $t$ in $q=e^{-4 t}$. The Fourier transform of $Z_{D B}$ is given by

$$
i \int d x^{0} e^{i E x^{0}} q^{-\frac{1}{4} E^{2}} Z_{D B}=\frac{\pi \lambda^{-i E}}{\sinh \pi E} \sum_{k=0}^{\infty} \frac{(i E)^{k}}{k !}\left[\chi_{E+2 i k}(q)-\chi_{E+2 i(k+1)}(q)\right],
$$

where $\chi_{E}(q)$ is the character in the closed string channel

$$
\chi_{E}(q)=\frac{1}{\eta(q)} q^{-\frac{1}{4} E^{2}} .
$$

For simplicity, we ignored the delta-fucntion $\delta(E)$ discussed in section 2.5. After all, the annulus amplitude between $\left\langle B_{f}\right|$ and $|B\rangle$ is given by

$$
\left\langle B_{f}\left|q^{\frac{1}{2}\left(L_{0}+\widetilde{L}_{0}\right)-\frac{1}{24}}\right| B\right\rangle=\int \frac{d E}{2 \pi} \frac{\pi^{2}}{\sinh ^{2} \pi E} \sum_{k=0}^{\infty} \frac{(i E)^{k}}{k !}\left[\chi_{E+2 i k}(q)-\chi_{E+2 i(k+1)}(q)\right] .
$$


The integral over $E$ is not well-defined in this expression. As discussed in [19], to see the behavior of this integral it is useful to write $\chi_{E}(q)$ in terms of the non-oscillating open string character

$$
\chi_{E}(q)=\sqrt{\frac{\pi}{2}} \int_{-\infty}^{\infty} d \nu e^{\pi E \nu} \chi_{i \nu}\left(q_{o p}\right)
$$

where $q$ and $q_{o p}$ are the modulus in the closed string channel and the open string channel, respectively:

$$
q=e^{-4 t}, \quad q_{o p}=e^{-\pi^{2} / t}
$$

Then, the amplitude (4.24) in the open string channel is written as

$$
\begin{aligned}
& \left\langle B_{f}\left|q^{\frac{1}{2}\left(L_{0}+\widetilde{L}_{0}\right)-\frac{1}{24}}\right| B\right\rangle \\
= & \int \frac{d E d \nu}{2 \sqrt{2 \pi}} \frac{\pi^{2}}{\sinh ^{2} \pi E} \chi_{i \nu}\left(q_{o p}\right) \sum_{k=0}^{\infty} \frac{(i E)^{k}}{k !}\left[e^{\pi \nu(E+2 i k)}-e^{\pi \nu(E+2 i(k+1))}\right] \\
= & \int \frac{d E d \nu}{2 \sqrt{2 \pi}} \frac{\pi^{2}}{\sinh ^{2} \pi E} \chi_{i \nu}\left(q_{o p}\right)\left(1-e^{2 \pi i \nu}\right) \exp \left(\pi \nu E+i E e^{2 \pi i \nu}\right)
\end{aligned}
$$

The singularity at $E=0$ can be taken care of by subtracting the double pole $1 / E^{2}$ [19], or, by taking the pricipal value. The factor $1-e^{2 \pi i \nu}$ in (4.27) vanishes when $\nu$ is integer, which is the position of the poles in the corresponding expression of $\left\langle B_{f}\left|q^{\frac{1}{2}\left(L_{0}+\widetilde{L}_{0}\right)-\frac{1}{24}}\right| B_{f}\right\rangle$ [19]. Moreover, the large $E$ contribution is suppressed because of the oscilatory factor $e^{i E e^{2 \pi i \nu}}$. Therefore, the incorporation of the contribution from $\left|B_{0}^{\prime}\right\rangle$ qualitatively changes the analytic structure of the amplitude.

\section{Discussion}

We have shown that the contribution from the exponentially growing couplings to the timelike oscillators can be resummed in the annulus amplitude between $|B\rangle$ and $\left|B_{f}\right\rangle$. Although this result is suggestive, we cannot draw any conclusion about the amplitude between two $|B\rangle$ 's because we don't know the explicit form of the off-diagonal integral $Z_{N, M}$ with $N \neq M$.

To see whether the resummation in (3.5) is possible, it would be useful to find the underlying integrable structure of grand canonical partition function as in [42]. It would also be important to understand the physical meaning of the appearance of the superalgebra $u(N \mid M)$. In the superstring theory, the supergroup $U(N \mid M)$ naturally appears in the system of $N$ D-branes and $M \overline{\mathrm{D}}$-branes 43 . However, in our case, we are discussing 
the D-brane in the bosonic string theory and the $N$ and $M$ are the number of insertion of tachyon operators. The fermionic nature of the eigenvalues in unitary matrix integral might be related to the appearance of superalgebra. It is also interesting to generalize our analysis to the boundary state of unstable D-branes in superstring theory along the line of 10 .

\section{Acknowledgments}

I would like to thank N. Constable, M. Gutperle, M. Jinzenji, F. Larsen, H. Liu, E. Martinec, and M. Wakimoto for helpful discussions. This work was supported in part by DOE grant DE-FG02-90ER40560.

\section{Appendix A. Proof of $(4.17 a, b)$}

In this appendix, we give a proof of $(4.17 a, b)$ by taking a derivative of the Jacobi's triple product identity. Let us first consider (4.17a). Notice that the summation in the r.h.s of $(4.17 \mathrm{~d})$ can be extended to the whole integer

$$
\sum_{k=0}^{\infty}(-1)^{k}(k+1)^{2} q^{k(k+2)}=\frac{1}{2} \sum_{k \in \mathbf{Z}}(-1)^{k}(k+1)^{2} q^{k(k+2)} .
$$

This sum is obtained as a limit of the following Jacoi's triple product identity

$$
\begin{aligned}
\sum_{k \in \mathbf{Z}}(-1)^{k} a^{(k+1)^{2}} q^{k(k+2)} & =a \prod_{n=1}^{\infty}\left(1-a^{2 n} q^{2 n}\right)\left(1-a^{2 n-3} q^{2 n-3}\right)\left(1-a^{2 n+1} q^{2 n+1}\right) \\
& =-q^{-1} \prod_{n=1}^{\infty} \frac{1-a^{n} q^{n}}{1+a^{n} q^{n}} .
\end{aligned}
$$

Here we used the well-known relation

$$
\prod_{n=1}^{\infty}\left(1-x^{2 n-1}\right)=\prod_{n=1}^{\infty} \frac{1}{1+x^{n}}
$$

Substituting $a=1+\varepsilon$ into (A.2), and comparing the order $\varepsilon$ term on both sides, we have

$$
\begin{aligned}
\sum_{k \in \mathbf{Z}}(-1)^{k}(k+1)^{2} q^{k(k+2)} & =\prod_{n=1}^{\infty} \frac{1-q^{n}}{1+q^{n}} \sum_{m=1}^{\infty}\left(\frac{m q^{m-1}}{1-q^{m}}+\frac{m q^{m-1}}{1+q^{m}}\right) \\
& =2 \prod_{n=1}^{\infty} \frac{1-q^{n}}{1+q^{n}} \sum_{k=0}^{\infty} \frac{q^{2 k}}{\left(1-q^{2 k+1}\right)^{2}} .
\end{aligned}
$$


Eq.(4.17a) follows from (A.1) and (A.4).

Next consider (4.17b). As in (A.1), we first extend the range of summation

$$
\sum_{k=0}^{\infty}(-1)^{k}(k+1)(k+2)(2 k+3) q^{k(k+3)}=\frac{1}{2} \sum_{k \in \mathbf{Z}}(-1)^{k}(k+1)(k+2)(2 k+3) q^{k(k+3)} .
$$

Then writing the Jacobi's identity

$$
\sum_{k \in \mathbf{Z}}(-1)^{k} a^{k^{2}} b^{k} q^{k(k+3)}=\prod_{n=1}^{\infty}\left(1-a^{2 n} q^{2 n}\right)\left(1-a^{2 n-1} b q^{2 n+2}\right)\left(1-a^{2 n-1} b^{-1} q^{2 n-4}\right)
$$

and expanding the both sides around $a=b=1$, we find

$$
\begin{aligned}
\sum_{k \in \mathbf{Z}}(-1)^{k} q^{k(k+3)} & =0 \\
\sum_{k \in \mathbf{Z}}(-1)^{k} k q^{k(k+3)} & =-q^{-2} \prod_{n=1}^{\infty}\left(1-q^{2 n}\right)^{3}, \\
\sum_{k \in \mathbf{Z}}(-1)^{k} k^{2} q^{k(k+3)} & =3 q^{-2} \prod_{n=1}^{\infty}\left(1-q^{2 n}\right)^{3} \\
\sum_{k \in \mathbf{Z}}(-1)^{k} k^{3} q^{k(k+3)} & =\left(-7 q^{-2}+\sum_{k=0}^{\infty} \frac{6 q^{2 k}}{\left(1-q^{2 k+2}\right)^{2}}\right) \prod_{n=1}^{\infty}\left(1-q^{2 n}\right)^{3} .
\end{aligned}
$$

From (A.7) and (A.3), one can easily see that (4.17b) is true. 


\section{References}

[1] M. Gutperle and A. Strominger, "Spacelike branes," JHEP 0204, 018 (2002) hepth/0202210].

[2] A. Sen, "Rolling tachyon," JHEP 0204, 048 (2002) [hep-th/0203211.

[3] A. Sen, "Tachyon matter," JHEP 0207, 065 (2002) hep-th/0203265].

[4] A. Sen, "Field theory of tachyon matter," Mod. Phys. Lett. A 17, 1797 (2002) hepth/0204143].

[5] A. Sen, "Time evolution in open string theory," JHEP 0210, 003 (2002) hepth/0207105].

[6] A. Sen, "Time and tachyon," hep-th/0209122.

[7] P. Mukhopadhyay and A. Sen, "Decay of unstable D-branes with electric field," JHEP 0211, 047 (2002) hhep-th/0208142].

[8] A. Strominger, "Open string creation by S-branes," hep-th/0209090.

[9] B. Chen, M. Li and F. L. Lin, "Gravitational radiation of rolling tachyon," JHEP 0211, 050 (2002) hep-th/0209222.

[10] F. Larsen, A. Naqvi and S. Terashima, "Rolling tachyons and decaying branes," JHEP 0302, 039 (2003) hep-th/0212248.

[11] M. Gutperle and A. Strominger, "Timelike Boundary Liouville Theory," Phys. Rev. D 67, 126002 (2003) hep-th/0301038.

[12] A. Maloney, A. Strominger and X. Yin, "S-brane thermodynamics," [hep-th/0302146].

[13] T. Okuda and S. Sugimoto, "Coupling of rolling tachyon to closed strings," Nucl. Phys. B 647, 101 (2002) [hep-th/0208196].

[14] S. J. Rey and S. Sugimoto, "Rolling tachyon with electric and magnetic fields: Tduality approach," Phys. Rev. D 67, 086008 (2003) hep-th/0301049].

[15] S. J. Rey and S. Sugimoto, "Rolling of modulated tachyon with gauge flux and emergent fundamental string," Phys. Rev. D 68, 026003 (2003) [hep-th/0303133

[16] N. Lambert, H. Liu and J. Maldacena, "Closed strings from decaying D-branes," hepth/0303139].

[17] D. Gaiotto, N. Itzhaki and L. Rastelli, "Closed strings as imaginary D-branes," hepth/0304192].

[18] N. R. Constable and F. Larsen, "The rolling tachyon as a matrix model," JHEP 0306, 017 (2003) hep-th/0305177.

[19] J. L. Karczmarek, H. Liu, J. Maldacena and A. Strominger, "UV finite brane decay," hep-th/0306132.

[20] Y. Sugawara, "Thermal Partition Functions for S-branes," JHEP 0308, 008 (2003) hep-th/0307034.

[21] A. Sen, "Open-closed duality at tree level," hep-th/0306137.

[22] A. Sen, "Open-closed duality: Lessons from matrix model," hep-th/0308068. 
[23] V. Fateev, A. B. Zamolodchikov and A. B. Zamolodchikov, "Boundary Liouville field theory. I: Boundary state and boundary two-point function," hep-th/0001012.

[24] C. G. Callan, I. R. Klebanov, A. W. Ludwig and J. M. Maldacena, "Exact solution of a boundary conformal field theory," Nucl. Phys. B 422, 417 (1994) [hep-th/9402113].

[25] M. R. Gaberdiel, A. Recknagel and G. M. Watts, "The conformal boundary states for SU(2) at level 1," Nucl. Phys. B 626, 344 (2002) hep-th/0108102.

[26] A. Strominger and T. Takayanagi, "Correlators in timelike bulk Liouville theory," hep-th/0303221.

[27] V. Schomerus, "Rolling tachyons from Liouville theory," hep-th/0306026.

[28] J. Polchinski, "String Theory," volume I, Cambridge University Press, 1998.

[29] M. R. Douglas, "Conformal field theory techniques in large N Yang-Mills theory," hep-th/9311130.

[30] D. J. Gross and E. Witten, "Possible Third Order Phase Transition In The Large N Lattice Gauge Theory," Phys. Rev. D 21, 446 (1980).

[31] M. Goulian and M. Li, "Correlation Functions In Liouville Theory," Phys. Rev. Lett. 66, 2051 (1991).

[32] S. Cordes, G. W. Moore and S. Ramgoolam, "Lectures on 2-d Yang-Mills theory, equivariant cohomology and topological field theories," Nucl. Phys. Proc. Suppl. 41, 184 (1995) hep-th/9411210.

[33] J. McGreevy, J. Teschner and H. Verlinde, "Classical and quantum D-branes in 2D string theory," hep-th/0305194.

[34] M. R. Douglas, "Chern-Simons-Witten theory as a topological Fermi liquid," hepth/9403119].

[35] V. G. Kac, "Infinite-dimensional Lie algebras," 3rd edition, Cambridge University Press, 1990.

[36] I. G. Macdonald, "Affine root systems and Dedekind's $\eta$-function," Invent. Math. 15, 91 (1972).

[37] M. Jinzenji and T. Sasaki, " $N=4$ supersymmetric Yang-Mills theory on orbifold $T^{4} / Z_{2}$ : Higher rank case," JHEP 0112, 002 (2001) hep-th/0109159.

[38] I. G. Macdonald, "Symmetric Functions and Hall Polynomials," 2nd edition, Oxford University Press, 1995.

[39] V. G. Kac and M. Wakimoto, "Integrable Highest Weight Modules Over Affine Superalgebras And Number Theory," hep-th/9407057.

[40] Y. Matsuo, "Character Formula Of $c<1$ Unitary Representation Of N=2 Superconformal Algebra," Prog. Theor. Phys. 77, 793 (1987).

[41] D. Zagier, "A proof of the Kac-Wakimoto affine denominator formula for the strange series," Math. Res. Lett. 7, 597 (2000).

[42] V. Kazakov, I. K. Kostov and D. Kutasov, "A matrix model for the two-dimensional black hole," Nucl. Phys. B 622, 141 (2002) [hep-th/0101011].

[43] C. Vafa, "Brane/anti-brane systems and $\mathrm{U}(\mathrm{N} \mid \mathrm{M})$ supergroup," hep-th/0101218. 